\title{
Erratum: IL-1 is a critical regulator of group 2 innate lymphoid cell function and plasticity
}

Yoichiro Ohne, Jonathan S Silver, LuAnn Thompson-Snipes, Magalie A Collet, Jean Philippe Blanck, Brandi L Cantarel, Alan M Copenhaver, Alison A Humbles \& Yong-Jun Liu

Nat. Immunol.; doi:10.1038/ni.3447; corrected online 29 April 2016

In the version of this article initially published online, the interferon (IFN- $\alpha$ ) in the fourth sentence of the abstract is incorrect. That section should read "the locus encoding interferon- $\gamma($ IFN- $\gamma)$...." Also, sentence two in paragraph three of the introduction includes a typographical error ("zand"); that should read "IL-18 and IL-1..." The errors have been corrected for the print, PDF and HTML versions of this article.

\section{Erratum: A TRAF-like motif of the inducible costimulator ICOS controls development of germinal center $\mathrm{T}_{\mathrm{FH}}$ cells via the kinase TBK1}

Christophe Pedros, Yaoyang Zhang, Joyce K Hu, Youn Soo Choi, Ann J Canonigo-Balancio, John R Yates III, Amnon Altman, Shane Crotty \& Kok-Fai Kong

Nat. Immunol.; doi:10.1038/ni.3463; corrected online 20 May 2016

In the version of this article initially published online, in the keys in Figure 3i-l, the Icos-specific shRNA was incorrectly designated 'sh/cos'. The correct designation is 'shIcos'. The error has been corrected for the print, PDF and HTML versions of this article.

\section{Erratum: Inflammatory triggers associated with exacerbations of COPD orchestrate plasticity of group 2 innate lymphoid cells in the lungs}

Jonathan S Silver, Jennifer Kearley, Alan M Copenhaver, Caroline Sanden, Michiko Mori, Li Yu, Gretchen Harms Pritchard, Aaron A Berlin, Christopher A Hunter, Russell Bowler, Jonas S Erjefalt, Roland Kolbeck \& Alison A Humbles

Nat. Immunol.; doi:10.1038/ni.3443; corrected online 5 May 2016

In the version of this article initially published online, the units in the vertical axis for Figure 11 were incorrectly stated as '(fold)', and the figure lacked a key. The correct units are '(ng/ml; change from mock)'; the white bars are IL-5, the gray bars are IL-13, and the black bars are IFN- $\gamma$. Also, the lowest portions of the images in Figure 4i,j (including the inset in Figure 4j) were incorrectly cropped. The errors have been corrected for the print, PDF and HTML versions of this article.

\section{Erratum: Methyltransferase Dnmt3a upregulates HDAC9 to deacetylate the kinase TBK1 for activation of antiviral innate immunity.}

Xia Li, Qian Zhang, Yuanyuan Ding, Yiqi Liu, Dezhi Zhao, Kai Zhao, Qicong Shen, Xingguang Liu, Xuhui Zhu, Nan Li, Zhongyi Cheng, Guoping Fan, Qingqing Wang \& Xuetao Cao Nat. Immunol.; doi:10.1038/ni.3464; corrected online 9 June 2016

In the version of this article initially published online, the blot in Figure $7 \mathrm{~d}$ was a duplicate of the blot in Figure 7c. The error has been corrected in the print, PDF and HTML versions of this article. 